\title{
An investigation of the effects of osteoporosis, impact intensity and orientation on human femur injuries: a parametric finite element study
}

\author{
Ramin Shahbad', Mohsen Mortazavi', Fereshteh Alizadeh-Fard', Zeinab Mohammadi', Fatemeh Alavi', \\ Mohammed N. Ashtiani ${ }^{*}$ \\ 'Department of Aerospace, Faculty of Mechanical Engineering, Tarbiat Modares University, Tehran, Iran \\ ${ }^{2}$ Department of Physical Therapy, Faculty of Medical Sciences, Tarbiat Modares University, Tehran, Iran
}

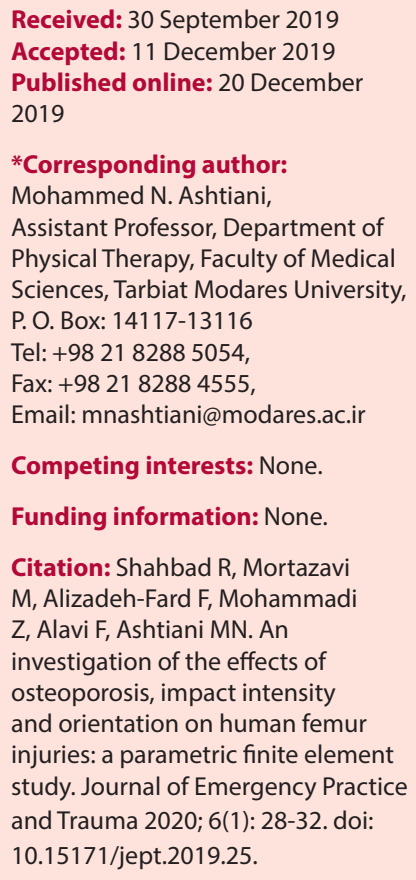

\begin{abstract}
Objective: Femur is the strongest, longest and heaviest bone in the human body. Due to the great importance of femur in human body, its injury may cause large numbers of disabilities and mortality. Considering various effective parameters such as mechanical properties, geometry, loading configuration, etc. can propel the study to the trustable results.

Methods: A 3D finite element model of the femur was subjected to different impact loading and orientations and also material properties. In addition to a reference healthy model of analysis, a total of 14 cases including four different loading conditions, six different bone density conditions and four different load orientations were considered. Results: Findings showed that the models with higher bone density cannot considerably reduce the stress under the impact loadings but porous models receive high mechanical stress which the bone prone to injury. The stress and displacement of the bone model received more values distributed through the femoral neck.

Conclusion: Porous bone models had greater stress values under an impact load. Higher and faster impacts may create multi-fracture breaks of the femur. The inferior femoral neck regions are the most vulnerable part in response to the impacts.

Keywords: Femur, Impact, Osteoporosis, Finite element
\end{abstract}

\section{Introduction}

The femur, the strongest bone in the human body, is the only bone in the upper leg which plays an important role in supporting human weight and allowing motion of the leg. The femur length is almost $26 \%$ of the human height and its major functions are to bear human weight and also handle the human balance during standing, walking and running (1). A femoral fracture usually occurs under large amounts of forces which can be the result of a car accident or high impact trauma $(2,3)$, leading to severe disabilities or mortality in humans. The incidence of these fractures varies globally, e.g. the city of Shiraz in Iran is reported to have the highest incidence of hip fractures in Asia (4). It is estimated that the worldwide number of hip fractures will be near 21.3 million by 2050 (5).

Although a growing segment of hip fracture is caused by decreasing bone strength due to osteoporosis, in people with normal bone strength, the most common causes of femur fractures include car accidents or fall from a height (3). It has been suggested that the loading characteristics and femoral features such as the femur geometrical characteristic and bone density (as an osteoporosis representative) are significant parameters that should be considered in order to predict the occurrence of hip fracture. Falling to the side has been identified to increase the risk by almost five times. The risk would further increase by more than twenty times if the impact occurs directly on the hip (6). Thus, in order to better predict the femoral fracture situation, it is needed to incorporate the information related to bone density (osteoporosis effect) and loading configurations (impact intensity and orientation).

Researchers have suggested various methods for noninvasive study of femoral injuries. Among these, the 
finite element method (FEM) based on the computed quantitative tomography (QCT) looms large in mind (7-11). Three-dimensional FE modelling is a common technique to predict femur stress and strain distribution under different loading configurations. The QCT techniques also provide an accurate $3 \mathrm{D}$ geometric model of bone. Sepehri et al conducted a research study on how different mechanical properties can affect the tibia bone stress analyses. It was found that the maximum stress would be seen in the case of the viscoelastic model of the tibia, while the minimum would occur in the case of transversely isotropic property (12). As one of the other loading conditions, femur bone progressive failure in sideway fall loading condition was investigated by Ridzwan et al using a CT-based FE model. The ultimate load and work needed for a complete femur fracture and also the fracture pattern were predicted (13). Ford et al using a 3D FEM model, indicated that the structural capacity of the femur is highly dependent on the direction of impact up to $26 \%$ ranging from $2090 \mathrm{~N}$ to $2820 \mathrm{~N}$ in different impact directions (14). Reddy and Kotiveerchari used a three-point bending test to simulate lateral unintended impacts in car accidents as one of the most common loading conditions to find the maximum allowable stress and deflection (3). Another experimental study was conducted to determine femur mechanical properties like strength and hardness during impact and bending testing on non-implanted and implanted bone (15).

In addition to the factors which have been examined by other researchers individually, the aim of this study is to investigate the effects of mechanical properties (osteoporosis), loading conditions (impact intensity) and impact direction (orientation) on human femur injuries using a parametric numerical finite element modeling.

\section{Methods}

To consider all the parameters of interest in this study, first, a base reference model was created. This reference model was the same for all conditions in geometry; however, material properties and boundary conditions were changed.

A realistic model of a young male femur was employed. The cloud point format of the CT-scanned data was used to reconstruct the $3 \mathrm{D}$ geometry of the femur. The distal epicondyle of the model was cut and then embedded in a cube of cement to exactly imitate the impact testing protocols (16). Two parts were then merged in order to remove relative displacements of the edges at the interface. The reference model had material properties associated with a normal healthy non-osteoporotic femur with the bone density equals to $1210 \mathrm{~kg} \cdot \mathrm{m}^{-3}$ (17). Besides the reference model, three cases with the bone densities higher and three cases lower than this level (to resemble with the osteoporosis) were also taken into account. In other words, the bone density $(\rho)$ was calculated using this equation: $\rho=1210 \pm \mathrm{n} \times 40\left(\right.$ in $\left.\mathrm{kg} \cdot \mathrm{m}^{-3}\right)$

where $\mathrm{n}=\{0,1,2,3\}$ and the case $\mathrm{n}=0$ is the reference density. The negative sign in eq. 1 represents the osteoporotic cases. The linear modulus of elasticity for the femur bone was calculated using below equation (17).

$\mathrm{E}=10200 \times \rho^{2.01}($ in $\mathrm{MPa})$

The cement block had modulus of elasticity equals with $17 \mathrm{GPa}$. The Poisson's ratios for bone and cement were 0.27 and 0.37 , respectively. All materials in the seven cases were linear elastic.

Loading: The reference model experienced an impact loading of $13 \mathrm{KN}$ during $10 \mathrm{~ms}$ normal to the midpoint of the femur head in the frontal plane with 60 degrees inclination to the femur shaft (reference loading). The impact was also applied to the model with different intensities as $16 \mathrm{KN}$ and duration of $100 \mathrm{~ms}$ (in fact, 2 magnitudes $\times 2$ durations $=4$ total intensity cases). In addition to the reference loading, the orientation of the impact load was also changed. Both in the transverse and frontal planes, the orientation included \pm 30 degrees to the reference loading. Therefore, five orientations were considered for application of the impact loads.

Boundary conditions: The degrees of freedom for side and bottom faces of the cement block were fully restricted to imitate the impact experiments.

Finite element: Each model was discretized to 58072 quadratic tetrahedral elements and 86605 nodes. The dynamic implicit solution criterion was considered in the finite elements analysis in Abaqus software (Abaqus, Dassault Systeme, version 6.10) to model the impact loads.

\section{Results}

Figure 1 shows the stress contours in a cut view of the models in all cases of analysis. The reference model was magnified in top left side to confer a better understanding of the results. Distribution of the higher stress values in majority of the cases was limited in the head up to the neck of the femur. Effects of bone densities (six bottom left panel, e-j) unveils that the osteoporotic bone models received roughly more distributed high-level stress. But the magnitudes remained the same. The different orientations of impact rather than the reference one (shown in bottom right panel, k-n) caused lower amount and distribution of the stress in the femur's head. Higher loading magnitudes in shorter time of application also increased and spread the stress not only through the head but also through the whole femur bone (top right panel, b-d).

The same panels for the displacement contours are also depicted in Figure 2. The most displacement contours were associated to the higher impact loads (top right panel, b-d). Except them, all cases received near zero displacements for regions beyond the femur's head. If the Mises criterion is assumed to determine the fracture, 


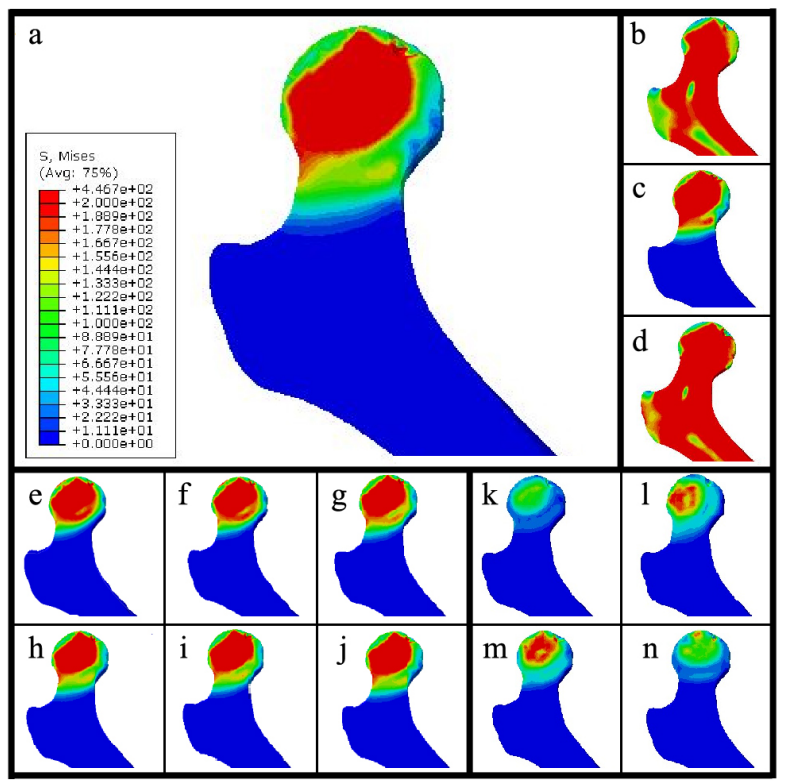

Figure 1. Stress contours of femur in a frontal cut view for (a) reference model, (b) higher impact load in a longer time, (c) reference impact load in a shorter time, (d) higher impact load in a shorter time, (e-g) osteoporotic bones deteriorated from right to left, $(\mathrm{h}-\mathrm{j})$ higher than normal bone densities increased from left to right, $(k)$ reference loading in transverse internal 30-degree orientation, (l) reference loading in frontal inferior 30-degree orientation, $(\mathrm{m})$ reference loading in frontal superior 30-degree orientation, (n) reference loading in transverse external 30-degree orientation.

the femur will be broken from the red highlighted regions in Figure 3. These regions located below the femur neck received stress values more than the Mises criterion in the reference model.

In addition, the stress and displacement due to the different loading and material conditions are plotted along a path that pass through the head, neck and the proximal epiphysis, as drawn in Figure 4. The stress values reveal a typical bimodal trend excepting the denser bone models. The highest displacement and stress belonged to the higher impact load. The oriented loadings in the transverse and frontal planes decreased the stress and displacement through the path.

\section{Discussion}

The purpose of this study was to investigate the effects of osteoporosis, impact intensity and impact orientation on the femur injuries. At first, a base reference model was considered for the study. Although the model had an identical geometry for each case, the material properties and boundary conditions were varied for each case.

Use of a parametric finite element model to inspect the vulnerability of the femur against the impact loads can present a larger number of outputs that is difficult and cost effective in ex vivo experiment. This study considered 13 different cases in addition to a reference model assumed as a healthy bone subjected to a normal impact imposed by the acetabular cavity reaction force on the femoral

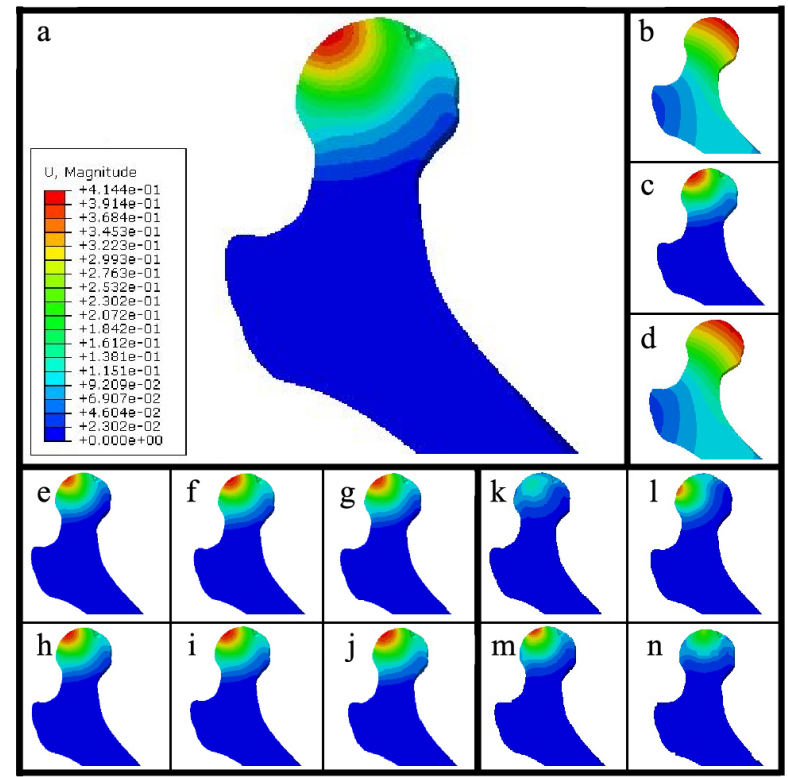

Figure 2. Displacement contours of femur in a frontal cut view for (a) reference model, (b) higher impact load in a longer time, (c) reference impact load in a shorter time, (d) higher impact load in a shorter time, $(e-g)$ osteoporotic bones deteriorated from right to left, $(h-j)$ higher than normal bone densities increased from left to right, $(k)$ reference loading in transverse internal 30-degree orientation, (I) reference loading in frontal inferior 30-degree orientation, $(\mathrm{m})$ reference loading in frontal superior 30-degree orientation, $(n)$ reference loading in transverse external 30-degree orientation.

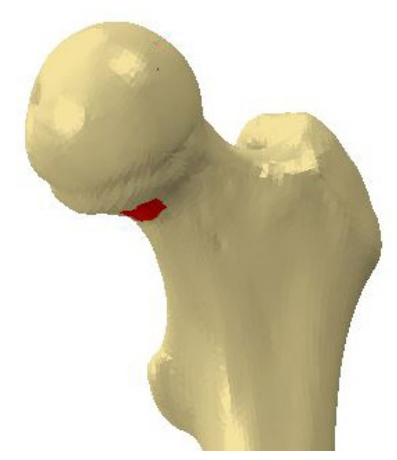

Figure 3. The most probable region of femur fracture subjected to the loadings used in the reference model with normal bone density. The stress in the red region exceeded the Mises criterion limit of failure.

head. The porosity of the bone that theoretically affects its Young's modulus was also decreased to represent the osteoporosis.

Distribution of the stress at the end of impact analysis (Figure 1) showed that in the reference model, the femur received higher values than its ultimate stresses. The ultimate stress for femur in tensile direction is about 135 $\mathrm{MPa}$, while for the compression direction it is about 205 $\mathrm{MPa}$ (18). The stresses due to the impact in this study exceeded these limits indicating that the bone will fail. Since the impact loading was dynamic, Figure 1 only showed the last frame of the analysis for all cases. The first region that received higher stress than the bone ultimate 

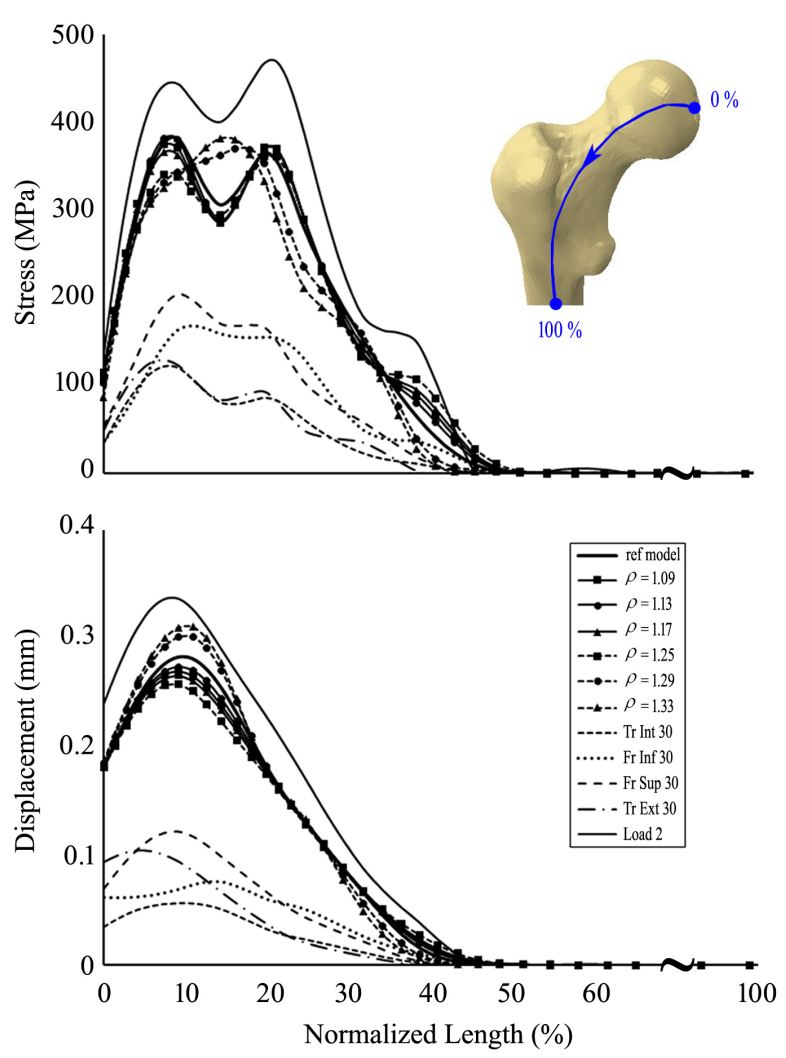

Figure 4. Variations of the stress and displacement for different material and loading cases.

stress threshold is shown in Figure 3. The inferior regions of femoral neck region were the first location that own failure stress under the impact load. This finding is in line with previous studies $(12,15)$. Although the nature of the impact load is roughly compressive to the neck, but the head and neck segment moved upward due to this loading direction. The displacement distribution through the same cut views (Figure 2) confirmed that the superior regions of the neck move upward to tense the inferiors. Therefore, it is predictable that the femur will be broken from the inferior regions of its neck. The calculated Mises criterion for the bone (Figure 3) also supports this finding. In order to compare the effects of porosity, impact magnitude and load direction, the last frames of the analysis were selected. The increase in load magnitude resulted in extreme stress values through the bone regions. Tippanagoudar and Krishna indicated that any increase in the load magnitude applied either in static or in impact mode causes the stress values of the tibia which is analogous to the femur (19). Ridzwan et al also showed that the increase in the impact intensity spreads the stress contours towards the mid-neck region (13). Application of such a higher load in a faster mode, deteriorated the case. In these conditions, all the bone regions received extreme stress and displacement values. The faster impact imposed higher energies to the bone that may result in fractures more than one region. The flow the stress through the diaphysis of the femur predicted possible multi-fractured femur.

The stress in the osteoporotic models was more distributed toward the neck at the last analysis frames. Lower bone elastic modulus in the porous models resulted in experiencing larger and more distributed stress and displacement contours. The denser bones, however, revealed no considerable reduction in stress and displacement values implying that the normal bone density behaves like the denser bone models in response to the impact loads.

Various impact orientations containing transverse internal $30^{\circ}$, transverse external $30^{\circ}$, frontal superior $30^{\circ}$ and frontal interior $30^{\circ}$ led to less femur head stress values and distribution. In all sideway fall loading configurations whose directions were lateral and posterolateral that affect femur greater trochanter, the high-stress ratio occurred on mid-neck and superior basicervical (14). In the frontal plane, the more upward forces caused more damages to the femur neck and increased the probability of failure from the inferior neck regions. The transverse loading, however, decreased the von Mises stresses through the femur and had a lower impact.

Evaluation of the stress along the defined path (Figure 4) showed a bimodal variation for the majority of the cases excepting the denser bone models. The first peak of the diagrams was related to the center of the femoral head which receives the highest amount of the stress. The second peak also belonged to the entrance of the neck which is narrower than the other regions. After that by moving toward the diaphysis, the stress values fell to vanish.

This study has some limitations. First, the material properties of the femur were considered as an isotropic elastic material. Some numerical studies on tibia showed that the viscoelastic models receive more amount of stress in a loading (12). Therefore, prospective studies can consider more realistic material properties for the bones. Second, the osteoporosis was included in the models merely by reducing the elastic modulus based on the reduced densities reported in the literature. It would be more realistic that the model considers composite material for the bone to directly assign the porosities.

\section{Conclusion}

The load impact due to the vehicle accident or fall can fail the femur from its inferior neck regions. Higher magnitude impacts may cause multi-fracture breaks. Porous cases of the femur receive higher and more distributed stress implying higher risks of fracture. The most harmful direction of loading to the femoral head is along the femoral neck. The inferior region of the femur is the first part that may break due to the tensile stresses.

Authors' contributions

MM prepared the FEM model from the CT-data. RS, FAF 
and ZM performed the FEM analysis. All first four authors wrote the manuscript. Design of the study, supervision and final check of the manuscript is done by FA and MNA.

\section{Ethical Issues}

This study used a finite element model and did not include human or animal subjects.

\section{References}

1. Kanis JA, Odén A, McCloskey EV, Johansson H, Wahl DA, Cooper C. A systematic review of hip fracture incidence and probability of fracture worldwide. Osteoporos Int 2012; 23(9): 2239-56. doi: 10.1007/s00198-012-1964-3.

2. Bojsen-Møller F, Tranum-Jensen J, Simonsen EB. Bevægeapparatets Anatomi. Copenhagen: Munksgaard; 2014.

3. Reddy AC, Kotiveerchari B. Simulation of femur bone fracture in car accident using CT scan data and finite element analysis. Int J Sci Res 2015; 4(11): 1805-7. doi: 10.21275/v4i11.nov151552.

4. Soveid M, Serati AR, Masoompoor M. Incidence of hip fracture in Shiraz, Iran. Osteoporos Int 2005; 16(11): 14126. doi: 10.1007/s00198-005-1854-z.

5. Gullberg B, Johnell O, Kanis JA. World-wide projections for hip fracture. Osteoporos Int 1997; 7(5): 407-13. doi: $10.1007 / \mathrm{pl} 00004148$.

6. Kannus $\mathrm{P}$, Sievänen $\mathrm{H}$, Palvanen $\mathrm{M}$, Järvinen T, Parkkari J. Prevention of falls and consequent injuries in elderly people. Lancet 2005; 366(9500): 1885-93. doi: 10.1016/ s0140-6736(05)67604-0.

7. Keyak JH. Improved prediction of proximal femoral fracture load using nonlinear finite element models. Med Eng Phys 2001; 23(3): 165-73. doi: 10.1016/s1350-4533(01)00045-5.

8. Keyak JH, Kaneko TS, Tehranzadeh J, Skinner HB. Predicting proximal femoral strength using structural engineering models. Clin Orthop Relat Res 2005; (437): 219-28. doi: 10.1097/01.blo.0000164400.37905.22.

9. Koivumäki JE, Thevenot J, Pulkkinen P, Kuhn V, Link TM, Eckstein F, et al. Ct-based finite element models can be used to estimate experimentally measured failure loads in the proximal femur. Bone 2012; 50(4): 824-9. doi: 10.1016/j. bone.2012.01.012.

10. Cody DD, Gross GJ, Hou FJ, Spencer HJ, Goldstein SA, Fyhrie DP. Femoral strength is better predicted by finite element models than QCT and DXA. J Biomech 1999; 32(10): 1013-20. doi: 10.1016/s0021-9290(99)00099-8.

11. Keyak JH, Rossi SA, Jones KA, Skinner HB. Prediction of femoral fracture load using automated finite element modeling. J Biomech 1998; 31(2): 125-33. doi: 10.1016/ s0021-9290(97)00123-1.

12. Sepehri B, Yazdi AA, Rouhi G. Comparison of the Effect of Different Mechanical Properties on the Stress Analysis of Tibia under Transversal Impact Loading Using Finite Element Method. In: Lim CT, Goh JCH, eds. 6th World Congress of Biomechanics (WCB 2010). August 1-6, 2010 Singapore. Berlin: Springer; 2010. p. 788-91. doi: 10.1007/978-3-642-14515-5_200.

13. Ridzwan MIZ, Pal B, Hansen UN. Finite element prediction of hip fracture during a sideways fall. International Scholarly and Scientific Research \& Innovation 2012; 6(10): 476-9.

14. Ford CM, Keaveny TM, Hayes WC. The effect of impact direction on the structural capacity of the proximal femur during falls. J Bone Miner Res 1996; 11(3):377-83.

15. Arun KV, Jadhav KK. Behaviour of human femur bone under bending and impact loads. Eur J Clin Biomed Sci 2016; 2(2): 6-13. doi: 10.11648/j.ejcbs.20160202.11.

16. Zdero R, Aziz MSR, Nicayenzi B. Quasi-static stiffness and strength testing of whole bones and implants. In: Zdero R, ed. Experimental Methods in Orthopaedic Biomechanics. Academic Press; 2017. p. 19-32. doi: 10.1016/B978-0-12803802-4.00002-0.

17. Keyak JH, Falkinstein Y. Comparison of in situ and in vitro CT scan-based finite element model predictions of proximal femoral fracture load. Med Eng Phys 2003; 25(9): 781-7. doi: 10.1016/s1350-4533(03)00081-x.

18. Reilly DT, Burstein AH. The elastic and ultimate properties of compact bone tissue. J Biomech 1975; 8(6): 393-405. doi: 10.1016/0021-9290(75)90075-5.

19. Tippanagoudar N, Krishna A. Finite element analysis of tibia bone. Int J Eng Sci Comput 2018; 8(12): 19534-7. 